\title{
First-principles modeling of interfaces between solids with large lattice mismatch: The prototypical $\mathrm{CoO}(111) / \mathrm{Ni}(111)$ interface
}

\author{
Sergiy Grytsyuk, Maxim V. Peskov, and Udo Schwingenschlögl* \\ Physical Science \& Engineering Division, KAUST, 23955-6900 Thuwal, Kingdom of Saudi Arabia \\ (Received 23 August 2012; revised manuscript received 8 November 2012; published 28 November 2012)
}

\begin{abstract}
In this work we investigate the $\mathrm{CoO}(111) / \mathrm{Ni}(111)$ interface by first-principles calculations, focusing on its structure and stability. To satisfy the approximate 5:6 ratio of the $\mathrm{CoO}$ and $\mathrm{Ni}$ lattice constants, we construct a supercell with $5 \times 5 \mathrm{Co}(\mathrm{O})$ and $6 \times 6 \mathrm{Ni}$ atoms per layer in the bulk regions. For the interface Ni layer and the adjacent Ni layer we consider different configurations and study the binding energy. We show for an ideal $\mathrm{CoO}$ interface terminated by $5 \times 5 \mathrm{O}$ atoms that the structure is more stable if there are $5 \times 5 \mathrm{Ni}$ atoms next to it instead of $6 \times 6$ as in the bulk. In addition, we observe that a transition layer with 31 or $33 \mathrm{Ni}$ atoms located between the interface $5 \times 5 \mathrm{Ni}$ and bulk $6 \times 6 \mathrm{Ni}$ layers (which partially reflects the structures of both these layers) enhances the stability of the $\mathrm{CoO} / \mathrm{Ni}$ interface. The electronic and magnetic modifications induced by the interface formation are discussed.
\end{abstract}

DOI: 10.1103/PhysRevB.86.174115

PACS number(s): 68.35.-p, 73.20.-r

\section{INTRODUCTION}

The development of magnetic devices based on coupling between a ferromagnet and an antiferromagnet (such as spin valves, ${ }^{1}$ metal-oxide-metal tunneling diodes, ${ }^{2}$ and magnetic recording media ${ }^{3}$ ) depends strongly on the interfacial magnetic structure. When approaching the nano and atomic scales, the physical and chemical properties of such devices are strongly affected by the structure of the interface and can be significantly modified. Even if the physical and chemical properties of the involved solids in their bulk form are known, investigation of the interface between them is a real challenge for experiments due to the small dimensions. An effective way to explore many aspects of such interfaces is opened by ab initio calculations.

Many reported $a b$ initio studies are related to interfaces with small lattice mismatch between the two solids. ${ }^{4}$ Modeling interfaces between compounds with significant lattice mismatch in first-principles investigations simply by attaching the materials to each other would give wrong results because of the induced strain. A large mismatch of the unit cell parameters, for example at an interface between face centered cubic and hexagonal close packed structures, can be dealt with by constructing a supercell that includes different numbers of unit cells of the compounds in the interface plane..$^{5-8}$ A main feature of such models is that the structures of the interface layers are chosen to be the same as in the bulk. This means that most of the interface atoms are not located in stable sites, for example hollow sites of the opposite interface layer, and have unrealistic distances to atoms on the other side of the interface. Of course, such a configuration is not stable and the question about the atomic density at the interface becomes eminent.

For our present investigation we choose the prototypical $\mathrm{CoO} / \mathrm{Ni}$ interface. This interface can be understood as a simplification of the $\mathrm{CoO} /$ permalloy interface, which is very attractive since the Neel temperature of $\mathrm{CoO}$ is close to room temperature and permalloy has a high permeability, low magnetostriction, and high anisotropic magnetoresistance. Extensive studies have been reported in the literature. ${ }^{9-17}$ From the theoretical point of view, the large lattice mismatch between $\mathrm{CoO}$ and $\mathrm{Ni}$ (about 21\%) can be addressed by constructing a supercell with $5 \times 5 \mathrm{CoO}$ and $6 \times 6 \mathrm{Ni}$ unit cells in contact with each other. Going beyond this approximation, we consider in the present work that the structures of the atomic layers around the interface can differ from the respective structures in the bulk regions of the supercell. We construct various possible structures using the hard sphere model and calculate the binding energy. Analyzing the distances between the atoms at the interface, we search for the most stable structure of the $\mathrm{CoO} / \mathrm{Ni}$ interface.

\section{COMPUTATIONAL DETAILS}

$A b$ initio calculations are performed in the framework of density functional theory using the plane-wave approach ${ }^{18-20}$ with the generalized gradient approximation $(\mathrm{GGA})^{21}$ and projector augmented wave pseudopotentials ${ }^{22,23}$ with the electronic configurations $\mathrm{O} 2 s^{2} 2 p^{4}$, Co $3 d^{8} 4 s^{1}$, and $\mathrm{Ni} 3 d^{9} 4 s^{1}$. We have verified that a plane-wave basis set with energies up to $400 \mathrm{eV}$ is accurate. Because of the size of our supercells (14.96 $\AA \times 34.44 \AA$ ) a $\Gamma$-point calculation is sufficient. We have verified for several cases that the energy difference with respect to a $2 \times 2 \times 1 k$ mesh is less than $0.01 \mathrm{eV}$ per atom. The density of states (DOS) is calculated with a Gaussian smearing of 0.05 $\mathrm{eV}$. To account for the localization of the $3 d$ orbitals in $\mathrm{CoO}$, we consider an onsite Coulomb interaction in the rotationally invariant Dudarev scheme $(\mathrm{GGA}+U)^{24-26}$ with an effective value of $U=6.1 \mathrm{eV}^{27,28}$ The $3 d$ orbitals of the Ni atoms at the interface are subject to strong Coulomb repulsion if they bond to $\mathrm{O}$ (similar to $\mathrm{NiO}$ ), while towards the bulk $\mathrm{Ni}$ we have instead free carriers (similar to bulk Ni). While the GGA works reasonably well for metals, it is known to underestimate the magnetic moments and band gaps in transitional metal oxides. The GGA $+U$ approach can overcome this problem for metal oxides, but is not appropriate for metals. To compensate for this inaccuracy in our calculations, we employ both the GGA and $\mathrm{GGA}+U$ methods for the interface $\mathrm{Ni}$ atoms and compare the results, whereas the other $\mathrm{Ni}$ atoms are always treated in the GGA. The effective interaction parameter for the interface $\mathrm{Ni}$ atoms is taken from bulk $\mathrm{NiO}(U=7 \mathrm{eV}){ }^{29}$ 


\section{INTERFACE MODELS}

$\mathrm{CoO}$ crystallizes in the $\mathrm{NaCl}$ structure type with the Co magnetic moments arranged antiferromagnetically (AFM-II order), while $\mathrm{Ni}$ follows the $\mathrm{Cu}$ structure type (cubic close packed) and shows ferromagnetism (FM order). To construct the $\mathrm{CoO} / \mathrm{Ni}$ interface we choose a (111) orientation for both $\mathrm{CoO}$ and $\mathrm{Ni}$, because this surface of $\mathrm{CoO}$ is not compensated and the exchange bias is maximal. ${ }^{13}$ The lattice mismatch is reduced to less than $1 \%$ as $5 \times 5 \mathrm{CoO}$ and $6 \times 6 \mathrm{Ni}$ unit cells are put in contact to each other. Perpendicular to the interface ( $z$ direction of the supercell) we place four $\mathrm{CoO}$ layers and five Ni layers as well as a vacuum spacing of $15 \AA$ thickness, which we find to be sufficient to avoid an interaction across the vacuum.

While in the bulk regions of our supercell the atoms build a close packed arrangement, the structure of the interface layers and the layers close to the interface may be different. We assume that the surface of $\mathrm{CoO}$ is terminated by a layer of $\mathrm{O}$ atoms that has no defects. We consider different atomic configurations for the first two layers of $\mathrm{Ni}$ atoms next to the interface (the numbers of $\mathrm{Ni}$ atoms in these layers are denoted by $x_{1}$ and $x_{2}$ ), focusing on the following two cases (Fig. 1): (i) The atoms in the first Ni layer occupy hollow sites of the adjacent $\mathrm{O}$ layer; see Fig. 1(b). The structure of the second $\mathrm{Ni}$ layer is not known, while the third Ni layer maintains a configuration of close packed $6 \times 6 \mathrm{Ni}$ atoms. (ii) The structure of the first Ni layer is not known, while the second Ni layer has the bulk structure; see Fig. 1(c). The layer with unknown structure located between layers of 25 and 36 close packed atoms is called transition layer. Cases (i) and (ii) describe the location of the transition layer, i.e., between 25 and $36 \mathrm{Ni}$ atoms in case (i) and between $25 \mathrm{O}$ and $36 \mathrm{Ni}$ atoms in case (ii). These structures are justified by the hard sphere model; see Fig. 2(a). The bonding around the interface is maximal if the atoms have the same distance to three neighbors on the other side of the interface (threefold hollow sites). Twofold bridge sites are less favorable and top sites are least favorable. ${ }^{5}$ We consider the possible structures of the transition layer for which each atom occupies at least one face centered cubic hollow site, as indicated in Fig. 2 by yellow triangles. The possibility that the

(a)



FIG. 1. (Color online) $($ a) $(5 \times 5) \mathrm{CoO} /(6 \times 6) \mathrm{Ni}$ interface. The vertical lines highlight the $\mathrm{Ni}$ and $\mathrm{CoO}$ unit cells. The structures of layers 1 and 2 (frist two Ni layers) are unknown with $x_{1}$ and $x_{2} \mathrm{Ni}$ atoms in these layers. (b) The atoms in layer 1 occupy hollow sites of the adjacent $\mathrm{O}$ layer. (c) The atoms in layer 2 occupy hollow sites of the adjacent Ni layer. interface stacking could deviate from the face centered cubic stacking sequence common to both $\mathrm{CoO}$ and $\mathrm{Ni}$ is not taken into account for simplicity.

For a better understanding, let us divide the interface area of the supercell into the regions I and II as indicated in Fig. 2(a). We first consider the case that all atoms in the transition layer occupy hollow sites of one of the surrounding layers; see Figs. 2(b) and 2(c). In this case the transition layer contains 25 or 36 close packed atoms. Such interfaces are expected to suffer from the nonuniform environments of the interface atoms, which are shifted from the energetically most favorable positions. The transition layer in Fig. 2(d) comprises 31 atoms. In region I they occupy hollow sites of the top layer and in region II hollow sites of the bottom layer. In contrast to the previous two models, here no top sites are occupied, which stabilizes this structure. In Fig. 2(e) the transition layer (containing 33 atoms) has more atoms on the hollow sites of the top layer than of the bottom layer, while in Fig. 2(f) the majority of the atoms in the transition layer (containing 28 atoms) occupy hollow sites of the bottom layer. We see that top sites can be completely avoided. Applying the introduced configurations of the transition layer and having two possibilities to locate it, we obtain seven structures with different numbers of $\mathrm{Ni}$ atoms in the first two layers; see Table I. It turns out that the $\mathrm{Ni}$ and $\mathrm{Co}$ atoms at the interface couple antiferromagnetically and the $\mathrm{Ni}$ atoms in the first two layers ferromagnetically for all structures under consideration.

\section{RESULTS}

To find the most stable structure among the models in Table I, we calculate the average binding energy for the $\mathrm{Ni}$ atoms in the first two layers (which contain $x_{1}+x_{2}$ atoms in total) as

$$
E_{b}=\frac{E_{\mathrm{CoO} / \mathrm{Ni}}-E_{\mathrm{CoO}}-E_{\mathrm{Ni}, \text { bulk-like }}}{x_{1}+x_{2}}-E_{\mathrm{Ni}, \text { atom }}
$$

where $E_{\mathrm{CoO} / \mathrm{Ni}}, E_{\mathrm{CoO}}, E_{\mathrm{Ni} \text {,bulk-like}}$, and $E_{\mathrm{Ni} \text {,atom }}$ denote the energies of the full supercell, a supercell with only the $\mathrm{CoO}$ region, a supercell with only the bulk-like $\mathrm{Ni}$ region, and one $\mathrm{Ni}$ atom, respectively. All these supercells have the same lattice parameters. Figure 3(a) shows that configurations (2) and (3) are most stable when we apply the GGA and GGA $+U$ methods for the $\mathrm{Ni}$ atoms at the interface, respectively. However, both these methods favor 25 atoms in the first $\mathrm{Ni}$ layer, i.e., the second $\mathrm{Ni}$ layer is the transition layer; compare with Fig. 1(b). We observe that the structure of this transition layer is different for the GGA and GGA $+U$ approaches: In the GGA the lowest energy is obtained when there are 31 atoms in the transition layer; see Fig. 2(c), while the GGA $+U$ approach favors 33 atoms; see Fig. 2(d). To understand which of the two methods describes the structural properties of the

TABLE I. Numbers of atoms in Ni layers 1 and 2, see Fig. 1, for the different models considered for the $\mathrm{CoO} / \mathrm{Ni}$ interface.

\begin{tabular}{llllllll}
\hline \hline Model & $(1)$ & $(2)$ & $(3)$ & $(4)$ & $(5)$ & $(6)$ & $(7)$ \\
\hline Layer 1 & 25 & 25 & 25 & 25 & 31 & 33 & 36 \\
Layer 2 & 28 & 31 & 33 & 36 & 36 & 36 & 36 \\
\hline \hline
\end{tabular}


(a)

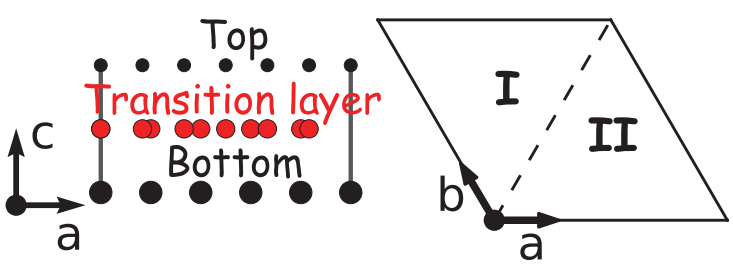

(b) 25 atoms in transition layer

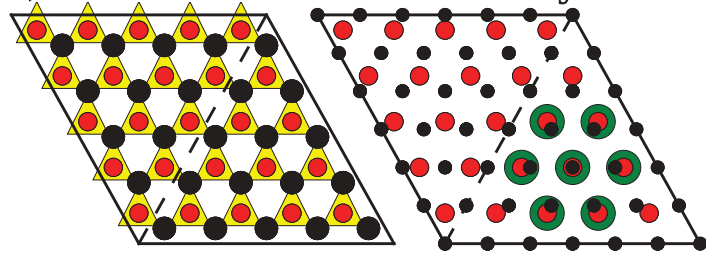

(c) 36 atoms in transition layer

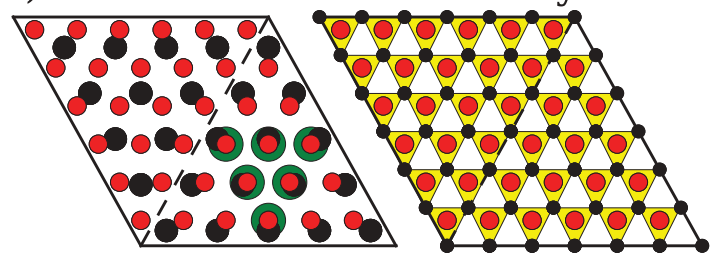

(d) 31 atoms in transition layer

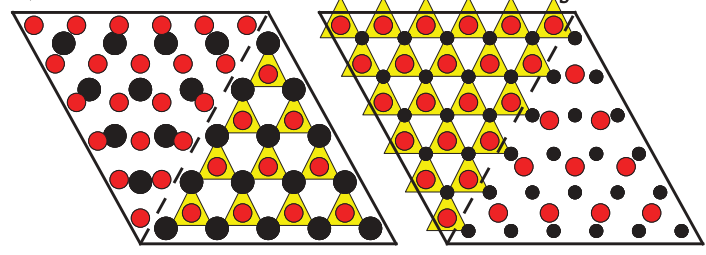

(e) 28 atoms in transition layer

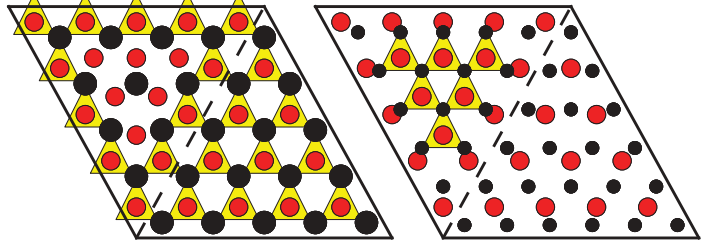

(f) 33 atoms in transition layer

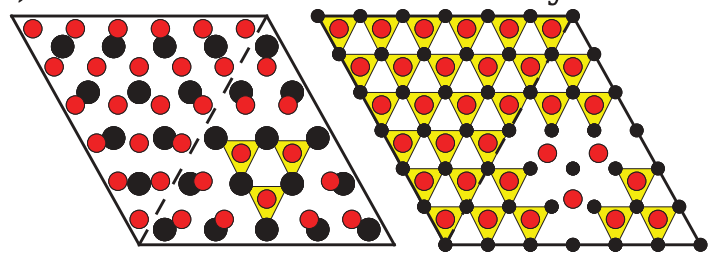

FIG. 2. (Color online) (a) The transition layer is located between a top layer with 25 and a bottom layer with 36 close packed atoms. (b)-(f) Possible structures of the transition layer. Yellow (green) background color indicates atoms on hollow (top) sites.

interface $\mathrm{Ni}$ atoms better, we have performed test calculations for bulk $\mathrm{Ni}$ and bulk $\mathrm{NiO}$ using different values of $U(0,3$, 5 , and $7 \mathrm{eV}$ ). Since we find that the lattice parameter of $\mathrm{NiO}$ does not vary significantly with $U$, the structure of the first $\mathrm{Ni}$ layer is essentially the same for the GGA and GGA $+U$ approaches. The obtained value of about $2.08 \AA$ is close to

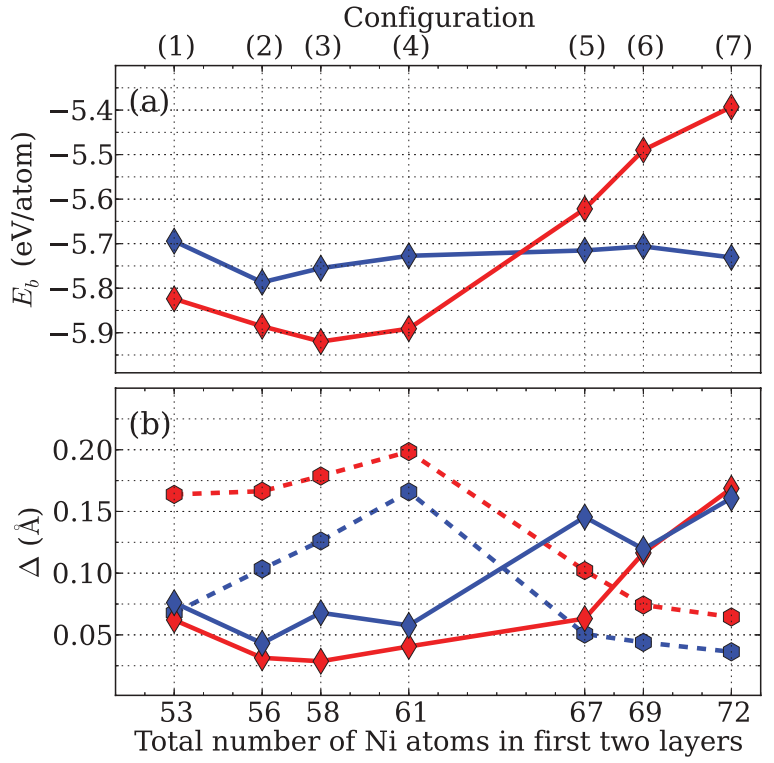

FIG. 3. (Color online) (a) Average binding energy per atom in layer 1 or 2 as calculated for the models of Table I. Blue (red) color represents GGA $(\mathrm{GGA}+U)$ results. (b) Average deviations from the bulk values of $\mathrm{Ni}-\mathrm{O}$ distances (solid) and of distances between $\mathrm{Ni}$ atoms in the two sublayers (dashed). the experimental value..$^{30,31}$ In contrast, the lattice parameter of bulk Ni decreases with increasing $U$ remarkably from 2.49 to $2.43 \AA$; see Fig. 4(b). Therefore, the reason for the different structures of the transition layer when the GGA and GGA $+U$ methods are applied to the interface $\mathrm{Ni}$ atoms is that the former method leads to larger $\mathrm{Ni}-\mathrm{Ni}$ distances.

To understand why the $(5 \times 5) \mathrm{CoO} /(6 \times 6) \mathrm{Ni}$ interface is most stable when there are 25 and $31 / 33 \mathrm{Ni}$ atoms in the first
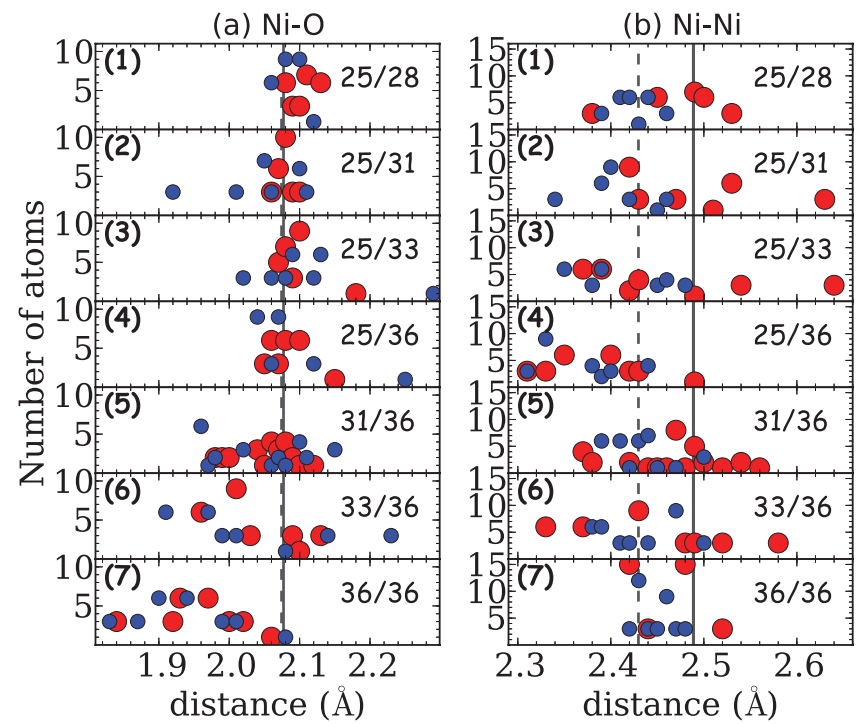

FIG. 4. (Color online) Distances between nearest neighbor atoms for models (1) to (7): (a) O and Ni, (b) Ni and Ni. Blue (red) color represents GGA $(\mathrm{GGA}+U)$ results. The vertical solid (dashed) lines give the bulk distances obtained for the GGA $(\mathrm{GGA}+U)$ method. 
two layers, we analyze the $\mathrm{Ni}-\mathrm{O}$ and $\mathrm{Ni}-\mathrm{Ni}$ distances. The further an atom is located away from the hollow site of the three atoms in the adjacent layer, the smaller is the binding energy. For this reason, we calculate the average deviations of the $\mathrm{Ni}-\mathrm{O}$ and $\mathrm{Ni}-\mathrm{Ni}$ distances from the values in bulk $\mathrm{NiO}$ and bulk $\mathrm{Ni}$, respectively. We have

$$
\Delta=\frac{\sum_{n=1}^{N} \sum_{i=1}^{3}\left|r_{\text {bulk }}-r_{i}^{n}\right|}{3 N},
$$

where $N$ is the total number of $\mathrm{O}$ or $\mathrm{Ni}$ atoms in the interface. The results are summarized in Fig. 3(b). We see that the lowest binding energy appears for the structures with the smallest deviations of the Ni-O distances from the bulk value, i.e., for configurations (1) to (4) with $25 \mathrm{Ni}$ atoms in the interface. In contrast, the influence of the Ni-Ni distances is small, which can be explained by the fact that metal-O bonds are stronger than metal-metal bonds. As a consequence, the $\mathrm{Ni}$ atoms at the interface replicate that structure of bulk $\mathrm{NiO}$ instead of that of bulk Ni. Another important detail that we can deduce from Fig. 3(b) is the following: While the number of $\mathrm{Ni}$ atoms in the first layer is the same for configurations (1) to (4), the stability of the interface (in terms of binding energy or distances) is determined by the structure of the transition layer. The structure is most stable when there are $31 / 33$ atoms in the transition layer which occupy partially the hollow sites of both adjacent layers.

In Figs. 4(a) and 4(b) we summarize the obtained distances between nearest neighbor $\mathrm{Ni}$ and $\mathrm{O}$ atoms as well as between nearest neighbor $\mathrm{Ni}$ atoms within the first two layers. We find that the average deviation of the $\mathrm{Ni}-\mathrm{O}(\mathrm{Ni}-\mathrm{Ni})$ distance from the bulk value $2.08 \AA(2.49 \AA)$ is minimal when there are 25 (36) Ni atoms in the interface. Because the binding energy depends strongly on the distance between the atoms, increasing the number of $\mathrm{Ni}$ atoms increases the binding energy for $\mathrm{Ni}-\mathrm{Ni}$ interaction but decreases it for $\mathrm{Ni}-\mathrm{O}$ interaction. If there are $36 \mathrm{Ni}$ atoms in the interface as in model (7), there are a few too-short Ni-O distances (about $1.83 \AA$ ), which is not realistic. This means that the structure of the Ni layer at the interface cannot be that of the bulk. If there are $25 \mathrm{Ni}$ atoms in the interface as in models (1) to (4), the structure of the transition layer has little influence on the Ni-O distances but results in significant deviations of the Ni-Ni distances: the more $\mathrm{Ni}$

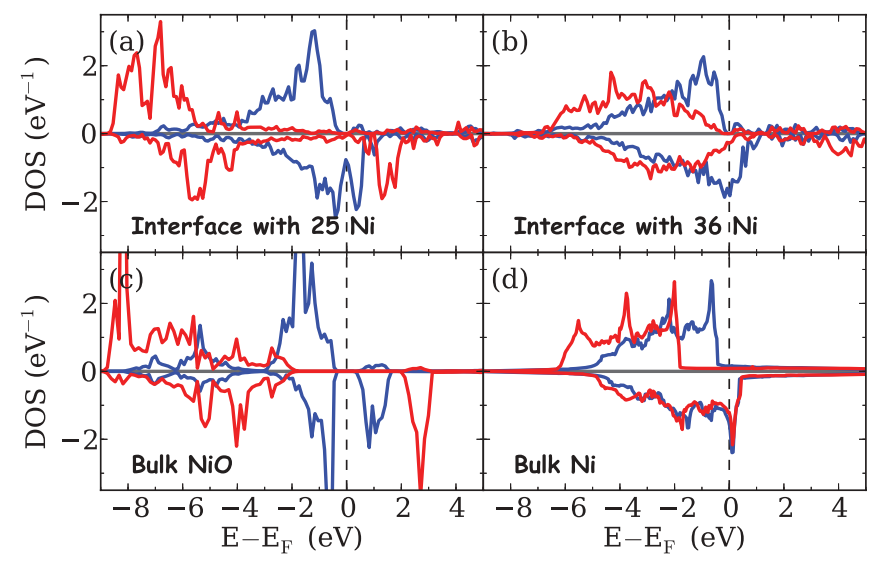

FIG. 5. (Color online) Ni DOS: Average over the Ni atoms in the interfaces with (a) 25 and (b) 36 atoms, respectively; (c) bulk NiO; and (d) bulk Ni. Blue (red) color represents GGA $(\mathrm{GGA}+U)$ results.

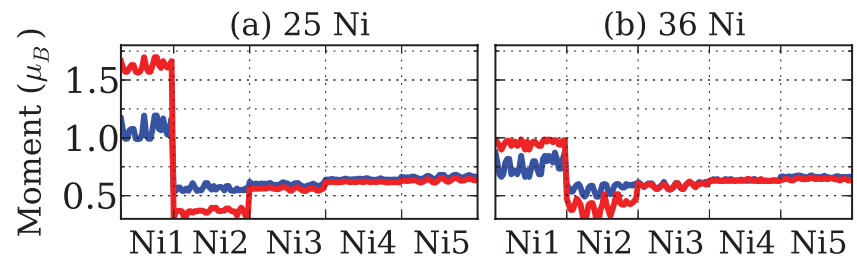

FIG. 6. (Color online) Magnetization profile of the Ni atoms for (a) 25 atoms [model (2)] and (b) 36 atoms [model (7)] in the interface. Blue (red) color represents GGA $(\mathrm{GGA}+U)$ results. The labels of the Ni layers refer to their distance to the interface.

atoms the bigger the deviations. For example, in model (4) we have a lot of short distances between $\mathrm{Ni}$ atoms (three times $2.31 \AA$ and nine times $2.33 \AA$ ), while for model (2) there are only three short distances of $2.34 \AA$. We notice that the GGA $+U$ method generally results in smaller variations of the Ni-O distances, whereas the GGA method gives better distances between the $\mathrm{Ni}$ atoms. As a consequence, Fig. 4 shows that for the GGA method model (2) is more stable than model (3), since the Ni-Ni distances deviate less from the bulk value. On the other hand, for the GGA $+U$ method model (3) is favored, since the $\mathrm{Ni}-\mathrm{O}$ distances are closer to the bulk value.

Our first-principles calculations clearly show that $\mathrm{Ni}$ atoms at the $\mathrm{O}$ terminated $\mathrm{CoO} / \mathrm{Ni}$ interface prefer the $\mathrm{O}$ hollow sites rather than the Ni hollow sites on the other side. How does this compare to experimental results? Experimentally, there is evidence for oxidation of the first metal layer. ${ }^{16}$ For this reason, we compare the electronic structures of the interface $\mathrm{Ni}$ atoms of models (4) and (7), which have 25 and 36 atoms in the interface, with the $\mathrm{Ni}$ atoms in bulk $\mathrm{Ni}$ and bulk $\mathrm{NiO}$; see Fig. 5. We find that the $\mathrm{Ni}$ electronic structures of models (1) to (4) are similar to that of bulk NiO. For 36 atoms each $\mathrm{Ni}$ is less oxidized than for 25 atoms, which leads to lower magnetic moments; see Fig. 6. According to this figure, the average $\mathrm{Ni}$ magnetic moment for the interface with 36 atoms is $0.76 \mu_{B}$ (GGA) or $0.95 \mu_{B}(\mathrm{GGA}+U)$ and for the interface with 25 atoms $1.07 \mu_{B}(\mathrm{GGA})$ or $1.62 \mu_{B}(\mathrm{GGA}+U)$. The observed oscillations of the magnetic moments are due to the different environments of the $\mathrm{Ni}$ atoms.

\section{v. CONCLUSION}

In this work we have dealt with the theoretical description of realistic $\mathrm{CoO}(111) / \mathrm{Ni}(111)$ interfaces. The large lattice mismatch between $\mathrm{CoO}$ and $\mathrm{Ni}$ has been addressed by a supercell approach with an interface of $5 \times 5 \mathrm{CoO}$ and $6 \times 6 \mathrm{Ni}$ unit cells. Assuming that the interface $\mathrm{Ni}$ atoms and $\mathrm{Ni}$ atoms in the next layer occupy at least one hollow site, we have analyzed possible interface structures. The calculated values of the binding energy show that the interface is most stable if there are $5 \times 5 \mathrm{Ni}$ atoms in contact with $5 \times 5 \mathrm{O}$ atoms, instead of the $6 \times 6$ atoms as expected from the bulk. The next Ni layer is a transition layer which interpolates between layers of $5 \times 5$ and $6 \times 6 \mathrm{Ni}$ atoms and enhances the stability of the interface if it partially reflects the structures of both these layers. The transition layer is found to optimally 
comprise 31 or $33 \mathrm{Ni}$ atoms. The obtained electronic structures show that the interface $\mathrm{Ni}$ atoms become more and more metallic and their magnetic moments decrease when the number of $\mathrm{Ni}$ atoms at the interface grows from 25 to 36 . The experimental evidence that the interface $\mathrm{Ni}$ atoms are oxidized is consistent with our result that there are only 25
$\mathrm{Ni}$ atoms in the interface. Our results for the prototypical $\mathrm{CoO}(111) / \mathrm{Ni}(111)$ interface demonstrate the possible effects of a varying atomic density, i.e., of the optimization of the chemical bonding, at interfaces between solids with significant lattice mismatch. In real interfaces there is in addition the possibility to have defects, which can modify the picture. *udo.schwingenschlog1@kaust.edu.sa, +966(0)544700080

${ }^{1}$ C. Fumeaux, J. Alda, and G. D. Boremann, Opt. Lett. 24, 1629 (1999).

${ }^{2}$ M. Ohkoshi, K. Tamari, M. Harada, S. Honda, and T. Kusuda, IEEE Trans. J. Magn. Jpn. 1, 37 (1985).

${ }^{3}$ Y. Fukuma, Le Wang, H. Idzuchi, S. Takahashi, S. Maekawa, and Y. Otani, Nat. Mater. 10, 527 (2011).

${ }^{4}$ S. Q. Wang and H. Q. Ye, Curr. Opin. Solid State Mater. Sci. 10, 26 (2006).

${ }^{5}$ R. Benedek, D. N. Seidman, M. Minkoff, L. H. Yang, and A. Alavi, Phys. Rev. B 60, 16094 (1999).

${ }^{6}$ R. Benedek, A. Alavi, D. N. Seidman, L. H. Yang, D. A. Muller, and C. Woodward, Phys. Rev. Lett. 84, 3362 (2000).

${ }^{7}$ X.-G. Wang and J. R. Smith, Phys. Rev. Lett. 95, 156102 (2005).

${ }^{8}$ S. Grytsyuk, F. Cossu, and U. Schwingenschlögl, Eur. Phys. J. B 85, 254 (2012).

${ }^{9}$ T. J. Moran, J. M. Gallego, and I. K. Schuller, J. Appl. Phys. 78, 1887 (1995).

${ }^{10}$ T. J. Moran and I. K. Schuller, J. Appl. Phys. 79, 5109 (1996).

${ }^{11}$ K. Takano, R. H. Kodama, A. E. Berkowitz, W. Cao, and G. Thomas, Phys. Rev. Lett. 79, 1130 (1997).

${ }^{12}$ K. Takano, R. H. Kodama, A. E. Berkowitz, W. Cao, and G. Thomas, J. Appl. Phys. 83, 6888 (1998).

${ }^{13}$ N. J. Gokemeijer, R. L. Penn, D. R. Veblen, and C. L. Chien, Phys. Rev. B 63, 174422 (2001).

${ }^{14}$ S. M. Zhou, S. J. Yuan, and L. Sun, J. Magn. Magn. Mater. 286, 211 (2005).

${ }^{15}$ K. W. Lin, F.-T. Lin, Y.-M. Tzeng, and Z.-Y. Guo, Eur. Phys. J. B 45, 237 (2005).
${ }^{16}$ S. Roy, C. Sanchez-Hanke, S. Park, M. R. Fitzsimmons, Y. J. Tang, J. I. Hong, D. J. Smith, B. J. Taylor, X. Liu, M. B. Maple, A. E. Berkowitz, C.-C. Kao, and S. K. Sinha, Phys. Rev. B 75, 014442 (2007).

${ }^{17}$ J. van Lierop, B. W. Southern, K.-W. Lin, Z.-Y. Guo, C. L. Harland, R. A. Rosenberg, and J. W. Freeland, Phys. Rev. B 76, 224432 (2007).

${ }^{18}$ G. Kresse and J. Hafner, Phys. Rev. B 47, 558 (1993).

${ }^{19}$ G. Kresse and J. Furthmüller, Phys. Rev. B 54, 11169 (1996).

${ }^{20}$ G. Kresse and D. Joubert, Phys. Rev. B 59, 1758 (1999).

${ }^{21}$ J. P. Perdew, K. Burke, and M. Ernzerhof, Phys. Rev. Lett. 77, 3865 (1996).

${ }^{22}$ P. E. Blöchl, Phys. Rev. B 50, 17953 (1994).

${ }^{23}$ G. Kresse and D. Joubert, Phys. Rev. B 59, 1758 (1999).

${ }^{24}$ V. I. Anisimov, I. V. Solovyev, M. A. Korotin, M. T. Czyzyk, and G. A. Sawatzky, Phys. Rev. B 48, 16929 (1993).

${ }^{25}$ V. I. Anisimov, J. Zaanen, and O. K. Andersen, Phys. Rev. B 44, 943 (1991).

${ }^{26}$ S. L. Dudarev, G. A. Botton, S. Y. Savrasov, C. J. Humphreys, and A. P. Sutton, Phys. Rev. B 57, 1505 (1998).

${ }^{27}$ T. Bredow and A. R. Gerson, Phys. Rev. B 61, 5194 (2000).

${ }^{28}$ F. Tran, P. Blaha, K. Schwarz, and P. Novák, Phys. Rev. B 74, 155108 (2006).

${ }^{29}$ O. Bengone, M. Alouani, P. Blöchl, and J. Hugel, Phys. Rev. B 62, 16392 (2000).

${ }^{30}$ W. Jauch, M. Reehuis, H. J. Bleif, F. Kubanek, and P. Pattison, Phys. Rev. B 64, 052102 (2001).

${ }^{31}$ W. Jauch and M. Reehuis, Phys. Rev. B 65, 125111 (2002). 